\title{
Beschäftigungs- und Lohnwirkungen von betrieblichen Reorganisationsprozessen
}

\author{
Lutz Bellmann
}

Angenommen: 25. Januar 2011 / Online publiziert: 8. Februar 2011

(C) Institut für Arbeitsmarkt- und Berufsforschung 2011

Zusammenfassung Betriebliche Reorganisationsprozesse sind durch verschiedene Bündel geschäftspolitischer, arbeitsorganisatorischer und personalpolitischer Maßnahmen, wie z. B. dem Abbau von Hierarchieebenen, Gruppenarbeit, job rotation, Weiterbildung, Anreizentlohnung und flexibler Arbeitszeiten, als Einzelmaßnahmen aber auch in ihrem $\mathrm{Zu}$ sammenwirken auf die Beschäftigung und Entlohnung von Erwerbstätigen auf unterschiedlichen Qualifikationsstufen gekennzeichnet. Die Verlagerung von Kompetenzen, Gruppenarbeit oder ein engerer Kundenkontakt erfordern Kommunikationsfähigkeit, Sozialkompetenz, Urteilsvermögen, Initiative und neben Kreativität auch die Eigenschaft, mit anderen zusammenzuarbeiten. Werden diese Fertigkeiten eher besser qualifizierten Mitarbeitern zugeschrieben, ist ein Unternehmen, das organisatorische Änderungen durchführt, bestrebt, ein entsprechend hohes Qualifikationsniveau zu etablieren. Insofern erscheint die Hypothese gerechtfertigt, dass betriebliche Reorganisationsmaßnahmen adverse Beschäftigungseffekte für Un- und Angelernte zur Folge haben.

Mit dem aus den Daten des IAB-Betriebspanels und der Sozialversicherungsdaten erstellten Linked-EmployerEmployee-Datensatz konnten die erwarteten positiven Auswirkungen des organisatorischen Wandels auf die betriebliche Arbeitsnachfrage belegt werden. Hinsichtlich des ,skill bias" des organisatorischen Wandels konnten allerdings durch Nachfrageschätzungen für einzelne Qualifikationsgruppen keine Hinweise gefunden werden. Auch bei den verallgemeinerten Leontief-Kostenfunktionen abgeleiteten Nachfragefunktionen für vier Qualifikationsgruppen, die als

Prof. Dr. L. Bellmann $(\bowtie)$

Institut für Arbeitsmarkt- und Berufsforschung, Bundesagentur für Arbeit, Regensburger Str. 104, 90478 Nürnberg, Deutschland e-mail: Lutz.Bellmann@iab.de „seemingly unrelated regressions“ (SUR) geschätzt werden, konnte der signifikante Einfluss der Variablen, die betriebliche Reorganisationsprozesse erfassen, nachgewiesen werden. Hinweise auf einen „skill bias“ des organisatorischen Wandels konnten wiederum nicht gefunden werden.

Außerdem zeigen sich positive Lohneffekte und stabilisierende Beschäftigungseffekte häufiger für Beschäftigte mit mittlerem oder hohem Ausbildungsstand. Dieses Ergebnis spricht für die Hypothese des qualifikationsverzerrenden organisatorischen Wandels. Eindeutig ihre Beschäftigung destabilisierende Effekte lassen sich für Un- und Angelernte allerdings mit der vorliegenden Untersuchung trotz der Anwendung alternativer methodischer Ansätze nicht feststellen.

Schlüsselwörter Arbeitsnachfrage - Qualifikatorische Lohn- und Beschäftigtenstruktur · Lohnpolitik

JEL Klassifikationen $\mathrm{J} 21 \cdot \mathrm{J} 23 \cdot \mathrm{J} 31 \cdot \mathrm{J} 38$

\section{Employment and wage effects of reorganisation of firms}

Abstract Organizational change is characterized by differ-
ent measures of business policy, work organization and per-
sonnel policy, e.g. delayering, teamwork, job rotation, fur-
ther training and education, incentive payment and flexible
working time. These measures work both, alone and in
combination, on employment and remuneration of employ-
ees of different skill levels. Relocation of responsibilities,
team work or close customer contact call for communica-
tion skills, social competence, judgment, initiative, creativ-
ity and even the individual capacity to work in teams. If
these skills are rather attributed to highly qualified work-
ers, an enterprise may be strived to establish a higher qual-
ification level after an organizational change. Against this 
background the hypothesis seems to be justified to assume, that organizational change causes adverse employment effects for low skilled workers.

Based on linked employer-employee data of the Institute of Employment Research (IAB) Nuremberg, built with the IAB Establishment Panel and the employment statistics register, positive effects of organizational change on labour demand can be shown. No empirical evidence could be found for a skill bias caused by organizational change in this respect. The demand functions, deducted from the generalized „Leontief"-cost function, have been estimated for four skill levels as seemingly unrelated regressions (SUR). Our results indicate a significant influence of these variables, which measure reorganizational change. Evidence for a skill bias of reorganizational measures could not be found.

Moreover positive effects on wages and stabilizing effects on employment appeared more often among middle or highly educated employees. This result corroborates the hypothesis of a skill biased organizational change. Clearly destabilizing effects on employment for low skilled workers could not be found in this study, despite using alternative methodological approaches.

\section{Einleitung}

In den letzten drei Jahrzehnten erlebten die westlichen Industrieländer weitreichende Veränderungen in den Arbeitsabläufen und den Entscheidungsprozessen innerhalb von Betrieben und Unternehmen. Diese betrieblichen Reorganisationsprozesse sind durch verschiedene Bündel geschäftspolitischer, arbeitsorganisatorischer und personalpolitischer Maßnahmen, wie z. B. dem Abbau von Hierarchieebenen, Gruppenarbeit, job rotation, Weiterbildung, Anreizentlohnung und flexibler Arbeitszeiten, als Einzelmaßnahmen aber auch in ihrem Zusammenwirken auf die Beschäftigung und Entlohnung von Erwerbstätigen auf unterschiedlichen Qualifikationsstufen gekennzeichnet. Oftmals werden diese Entwicklungen auf den technologischen Wandel und die Veränderungen im internationalen Handel zurückgeführt, außerdem wird von wichtigen Studien, wie die von Aoki (1990), Hammer und Champy (1993), Appelbaum und Bott (1994), Osterman (1994), Pfeiffer (1994), Lindbeck und Snower (1996, 2000) und Gallie et al. (1998) die Interdependenz und die gegenseitige Unterstützung der einzelnen Maßnahmen betont, so dass sogar gelegentlich von einer ,organisatorischen Revolution“ gesprochen wird. Bezüglich der Dynamik der Einführung der angesprochenen Dezentralisierung von Organisationsstrukturen finden Kirchner et al. (2008), dass ein Boom durch die Wirtschaftskrise 1992/93 in Deutschland ausgelöst wurde.

Diesen Maßnahmen werden neben Produktivitätseffekten auch positive Auswirkungen auf die betriebliche Ar- beitsnachfrage zugeschrieben, denn es werden Marktanteile gesichert oder sogar ausgebaut, was sich tendenziell beschäftigungsfördernd auswirkt. Nicht nur für Deutschland gibt es empirische Belege dafür, dass der mit der Einführung dieser Maßnahmen in den Betrieben sich vollziehende organisatorische Wandel - analog zu der Einführung neuer Technologien (Bellmann und Kölling 1997) nicht qualifikationsneutral erfolgt. Im Rahmen unseres Projektes wurden verschiedene Linked-Employer-Employee-Datensätze aus der Verknüpfung der Daten des IAB-Betriebspanels und der Daten der Beschäftigtenstatistik der Bundesagentur für Arbeit erstellt, um erstmals für einen verhältnismäßig langen Zeitraum von 1996 bis 2004 die Auswirkungen des organisatorischen Wandels auf die betriebliche Arbeitsnachfrage zu untersuchen.

Krugman (1994) hat daraufhin gewiesen, dass die Lohnund Beschäftigungseffekte des technologischen und des organisatorischen Wandels als zwei Seiten derselben Medaille zu betrachten sind. Das bedeutet, dass z. B. der Rückgang der Nachfrage nach un- und angelernten Beschäftigten durch eine entsprechende Lohnsenkung kompensiert werden müsste, um einen Abbau von Arbeitsplätzen für diese Beschäftigtengruppe zu vermeiden. Bestehen in dieser Situation Lohnrigiditäten oder werden tarifvertraglich die Löhne der Un- und Angelernten auf einem bestimmten relativen oder absoluten Abstand zu den Löhnen der Fachkräfte fixiert, ist ein Rückgang der Beschäftigung dieser Un- und Angelernten zu erwarten. Insofern würde bei einer Bestätigung der Hypothese eines „Skill bias“ des organisatorischen Wandels sich diese als Erklärung der vergleichsweise hohen Arbeitslosigkeit der Gruppe der Un- und Angelernten im Vergleich zu Personen mit Berufsausbildung und solchen mit Hochschulausbildung anbieten. In der Tat haben sich nach den Berechnungen von Biersack et al. (2008) die qualifikationsspezifischen Arbeitslosenquoten in Deutschland im Zeitraum 1975-2005 sehr unterschiedlich entwickelt. Dabei zeigt sich, dass die Arbeitslosenquote der Personen ohne Berufsabschluss 1975 noch bei 6,1\% lag, dann aber um mehr als das Vierfache auf 26,0 \% anstieg. Dazu erhöhte sich die Arbeitslosigkeit in allen anderen Qualifikationsgruppen weniger stark. So lag im Jahr 2005 die Arbeitslosenquote für alle Erwerbspersonen bei 11,8 \%. Erwerbspersonen mit abgeschlossener Lehre/Berufsausbildung waren hingegen $\mathrm{zu}$ 9,7 \% von Arbeitslosigkeit betroffen. Auch die Arbeitslosenquoten von Hochschul- und Fachhochschulabsolventen fielen mit 4,1\% deutlich geringer aus. In Ostdeutschland gab es eine ähnliche Strukturalisierung aber auf deutlich höherem Niveau.

Die ausgesprochen schlechten Arbeitsmarktchancen der Personen ohne beruflichen Bildungsabschluss zeigen sich auch bei den Anteilen der Nichterwerbspersonen. Während nach den Ergebnissen des Mikrozensus 2008 der Anteil der Nichterwerbspersonen im Alter von 55 bis 65 Jahren bei 
Akademikern 25,3 \% betrug, lag diese Quote bei Personen ohne beruflichen Bildungsabschluss bei 55,5\% und bei Personen mit Lehr-/Anlernausbildung bei 42,7 \% (Autorengruppen Bildungsberichterstattung 2010, 334).

Mit einem qualifikationsverzerrten organisatorischen Wandel ließe sich auch der Rückgang der Einfacharbeit in Deutschland erklären. Nach Berechnungen mit den Daten des IAB-Betriebspanels ist der Anteil der un- oder angelernten Beschäftigten sowie von Angestellten bzw. Beamten für einfache Tätigkeiten, die keine Berufsausbildung erfordern (so genannte Einfacharbeit) von $28 \%$ auf $23 \%$ der Erwerbstätigen und im Zeitraum von 2001 bis 2008 in Westdeutschland und in Ostdeutschland von $16 \%$ auf $13 \%$ zurückgegangen. Für Deutschland insgesamt ist der Anteil der Einfacharbeit von $26 \%$ auf $21 \%$ zurückgegangen. Entsprechend hat sich der Anteil der Facharbeit im genannten Zeitraum in Westdeutschland von $67 \%$ auf $72 \%$ und in Ostdeutschland von $78 \%$ auf $82 \%$ erhöht. Für Deutschland insgesamt gab es einen Anstieg des Anteils der Facharbeit um 5 Prozentpunkte von $69 \%$ auf $74 \%$ (Bellmann und Stegmaier 2010).

Die Entwicklung der Verteilung der Arbeitseinkommen in Westdeutschland über den verhältnismäßig langen Zeitraum von 1975 bis 2004 haben Dustmann et al. (2009) mit den Daten der IAB-Beschäftigtenstichprobe untersucht. Dabei konnten sie feststellen, dass die Lohnungleichheit deutlich zugenommen hat: In den 80er Jahren war die Zunahme der Lohnungleichheit auf die höheren Arbeitseinkommen beschränkt, während in den 90er Jahren sowohl untere als auch obere Teile der Einkommensverteilung betroffen waren. Die Autoren betonen für die Entwicklung im oberen Teil der Verteilung den Einfluss des technischen Wandels, während sie die Veränderungen im unteren Teil der Verteilung auf den Rückgang der Tarifbindung und eine Bevölkerungszunahme in Westdeutschland durch die Migration aus Mittel- und Osteuropa und Ostdeutschland zurückführen. Die Entwicklung in Westdeutschland erscheint damit der in den USA durchaus vergleichbar, wobei sie in Westdeutschland 10 Jahre später erfolgte.

Nachdem bereits als Einflussfaktoren für die Entwicklung der qualifikatorischen Beschäftigungs- und Lohnstruktur der technologische Wandel, die abnehmende Tarifbindung und die Zuwanderung von Un- und Angelernten erwähnt worden sind, soll im Folgenden die Rolle des organisatorischen Wandels näher betrachtet werden.

\section{Beiträge des Projekts zum Stand der Forschung}

2.1 Zur Hypothese des „skill biased organisational change“

Bei der Analyse der Lohn- und Beschäftigungseffekte von betrieblichen Reorganisationsprozessen werden verschiedene Qualifikationsstufen der Beschäftigten berücksichtigt. Es wird zwischen qualifizierten Beschäftigten und un- und angelernten Beschäftigten unterschieden. Zielsetzung war dabei, die Hypothese eines ,skill bias“ des organisatorischen Wandels zu untersuchen: Wird eine betriebliche Dezentralisierungsstrategie verfolgt, ist zudem der Wegfall mittlerer Hierarchieebenen wahrscheinlich. Deren Notwendigkeit kann dann durch den Einsatz von Informations- und Kommunikationstechnologien in Frage gestellt werden, wenn es so möglich ist, die Aufgaben der mittleren Führungsebene(n), also die Überwachung und Verteilung von Informationen, durch diese Technologien zu übernehmen (Lindbeck und Snower 1996). Dem gegenüber kann es für Unternehmen (z. B. bei der Einführung von Maßnahmen zur Qualitätssicherung) auch erforderlich werden, Spezialisten einzustellen. Ergeben sich im Unternehmen also durch betriebliche Reorganisationsmaßnahmen neue Aufgabenbereiche und neue Qualifikationserfordernisse, sollten diese sich in einer Erhöhung des Beschäftigungsniveaus niederschlagen, während eine „Straffung“ des Produktionsprozesses zu einem Personalabbau führen kann (Beckmann 2000). Es kann folglich keine einheitliche Wirkungsrichtung betrieblicher Reorganisationsprozesse auf die Anzahl der Beschäftigten festgestellt werden (Bellmann und Kohaut 1999). Zudem erfordert beispielsweise die Verlagerung von Kompetenzen, Gruppenarbeit oder ein engerer Kundenkontakt Kommunikationsfähigkeit, Sozialkompetenz, Urteilsvermögen, Initiative und neben Kreativität auch die Eigenschaft, mit anderen zusammenzuarbeiten. Zusätzlich kommt (dem Erwerb von) Fähigkeiten, die es ermöglichen, eine Vielzahl von Aufgaben zu erfüllen und Erfahrung aus einem Tätigkeitsfeld auf ein anderes zu übertragen, besondere Bedeutung zu (Lindbeck und Snower 2000). Werden diese Fertigkeiten eher besser qualifizierten Mitarbeitern zugeschrieben, ist ein Unternehmen, das organisatorische Änderungen durchführt, bestrebt, ein entsprechend hohes Qualifikationsniveau zu etablieren. Insofern erscheint es als gerechtfertigt, davon auszugehen, dass betriebliche Reorganisationsmaßnahmen adverse Beschäftigungseffekte für Un- und Angelernte zur Folge haben. Empirische Studien zum qualifikationsverzerrendem Effekt von organisatorischem Wandel beispielsweise für die USA, Frankreich, Italien und Großbritannien haben Greenan und Guellec (1998), Breshnahan et al. (2002), Caroli und van Reenen (2001) und Piva et al. (2003) vorgelegt.

\subsection{Beschäftigungswirkungen des organisatorischen Wandels}

Um dazu in einem ersten Schritt Arbeitsnachfragefunktionen unter Kontrolle für betriebliche Reorganisationsmaßnahmen mit einem dynamischen Panelansatz empirisch zu bestimmen, greifen Bellmann und Pahnke (2006) im Unterschied zu vergleichbaren Studien für Deutschland (siehe hierzu Bellmann und Schank 2000; Hujer et al. 2002; 
Bauer und Bender 2004; Bellmann et al. 2002; Kölling und Schank 2002) erstmals auf einen Panel-Datensatz für einen verhältnismäßig langen Zeitraum von 1996 bis 2004 zurück, der die Daten des IAB-Betriebspanels mit denen der Bundesagentur für Arbeit vorliegenden Sozialversicherungsdaten zusammenführt.

Um unterschiedlichen Zielsetzungen einzelner Reorganisationsmaßnahmen Rechnung zu tragen, werden diese auf Basis einer Faktorenanalyse von Alda und Bellmann (2002) in arbeits- bzw. produktionsstrukturverändernde Maßnahmen und sonstige Strukturveränderungen unterteilt. Diese Variablen werden durch Probit-Modelle instrumentiert, um die betriebliche Entscheidung, Reorganisationsmaßnahmen der genannten Gruppen einzuführen, angemessen im ökonometrischen Ansatz zu berücksichtigen. Mit dem GMMVerfahren (general method of moments) nach Arellano und Bond (1991) lassen sich dann dynamische Arbeitsnachfragefunktionen schätzen. Die so gewonnenen Ergebnisse zu den Auswirkungen organisatorischen Wandels auf die betriebliche Arbeitsnachfrage belegen die erwarteten positiven Effekte betrieblicher Reorganisationsmaßnahmen. Generell lassen sich kaum Hinweise auf einen ,skill bias“ des organisatorischen Wandels etwa durch Nachfrageschätzungen für einzelne Qualifikationsgruppen finden. Im Vergleich verschiedener Einzelmaßnahmen wird für West- und für Ostdeutschland deutlich, dass insbesondere die Reorganisation von Abteilungen adverse Beschäftigungseffekte zu Lasten Un- und Angelernter zeigt. Allerdings scheinen sich nicht alle von uns betrachteten Reorganisationsmaßnahmen gleichgerichtet auf die Beschäftigung einzelner Qualifikationsgruppen auszuwirken. So finden sich Hinweise darauf, dass die Einführung von Gruppenarbeit sich durchaus positiv auf das Beschäftigungsniveau Un- und Angelernter auswirken kann.

In einem weiteren Papier wurden von Addison et al. (2008) wiederum Linked-Employer-Employee-Daten genutzt, um die Nachfrage nach unterschiedlich qualifizierten Beschäftigten zu untersuchen. Es wurden aus verallgemeinerten Leontief-Kostenfunktionen Nachfragefunktionen für vier Qualifikationsgruppen abgeleitet und als ,,seemingly unrelated regressions“" (SUR) geschätzt, um die Erklärungskraft des technologischen wie auch organisatorischen Wandels vor dem Hintergrund der Entwicklungen im Bereich der Entlohnung und des internationalen Handels zu ermitteln. Dabei konnte in Übereinstimmung mit den Ergebnissen von Bellmann und Pahnke (2006) der signifikante Einfluss der Variablen, die betriebliche Reorganisationsprozesse erfassen, nachgewiesen werden. Hinweise auf einen „skill bias“ des organisatorischen Wandels konnten wiederum nicht gefunden werden. Allerdings identifizieren die berechneten Einkommensnachfrageelastizitäten Lohnrigiditäten in Deutschland als mögliche Ursache für die hohe Arbeitslosigkeit Un- und Angelernter.
2.3 Lohn- und Mobilitätseffekte des organisatorischen Wandels

Bellmann et al. (2008) haben die Auswirkungen der Reorganisationsmaßnahmen auf die Entlohnung untersucht. Es wurde geprüft, ob sich betriebliche Reorganisationsmaßnahmen überhaupt auf das betriebliche Lohnniveau auswirken, ob sich Unterschiede in der Entlohnung zwischen Qualifikationsgruppen zeigen und ob sich über die Entlohnung indirekte Effekte von Reorganisationsmaßnahmen auf die individuelle Mobilitätsentscheidung identifizieren lassen. Insbesondere wurde der Einfluss betrieblicher Reorganisationsmaßnahmen auf Betriebsaustritte, also auf die Stabilität von Beschäftigungsverhältnissen, betrachtet. Grundlage waren erneut die Linked-Employer-Employee-Daten für den Zeitraum 1996 bis 2004.

In den Fixed-Effects-Panelschätzungen für den Zeitraum von 1996 bis 2004 ergeben sich, getrennt für verschiedene Qualifikationsniveaus und Berufsgruppen, sehr differenzierte Ergebnisse. Es zeigt sich, dass für manche Beschäftigtengruppen die Einführung von Gruppenarbeit mit positiven Lohneffekten und stabilisierenden Effekten auf die Beschäftigung einhergeht. Die Reorganisation von Abteilungen, der vermehrte Zukauf von Produkten und die vermehrte Eigenfertigung führen bei einigen Gruppen zu negativen Lohneffekten und wenig stabilen Beschäftigungsverhältnissen. Weitere negative Lohneffekte konnten bei der Einrichtung von Einheiten mit eigener Kosten- und Ergebnisermittlung gefunden werden. Die Verbesserung der Qualitätssicherung führt bei verschiedenen Beschäftigtengruppen zu gegensätzlichen Lohneffekten.

Insgesamt finden sich positive Lohneffekte und stabilisierende Beschäftigungseffekte häufiger für Beschäftigte mit mittlerem oder hohem Ausbildungsstand. Dieses Ergebnis spricht für die Hypothese des qualifikationsverzerrenden organisatorischen Wandels. Eindeutig beschäftigungsdestabilisierende Effekte für Un- und Angelernte lassen sich allerdings mit der vorliegenden Untersuchung trotz der Anwendung alternativer methodischer Ansätze nicht feststellen.

Den Ergebnissen zufolge wirken Reorganisationsmaßnahmen meistens entweder auf die Löhne oder auf die externe Jobmobilität und die indirekte Wirkung auf die Beschäftigungsmobilität ist gering. Der Fall, dass Reorganisationsmaßnahmen nur auf die Beschäftigungsmobilität wirken, ist häufiger als der Fall, dass sie nur auf die Löhne wirken. Negative Lohneffekte sind seltener als positive Lohneffekte, während bei der Beschäftigungsmobilität Effekte in beide Richtungen vorliegen.

\section{Diskussion}

Die sich im Vergleich zu besser qualifizierten Erwerbspersonen während der letzten drei Jahrzehnte nicht nur in 
Deutschland verschlechternde Beschäftigungs- und Entlohnungssituation der Un- und Angelernten hat eine breite wissenschaftliche Diskussion über die möglichen Ursachen für diese Entwicklung ausgelöst. Die Auswirkungen des technologischen und des organisatorischen Wandels nehmen dabei eine zentrale Rolle ein. International vergleichende Studien sind in diesem Zusammenhang von besonderer Bedeutung, weil der Zugang zu modernen Technologien weltweit als gegeben betrachtet werden kann und zumindest in den entwickelten Ländern vergleichbare betriebliche Organisationskonzepte verwendet werden. Weiterhin können empirisch ermittelte Unterschiede bei den Auswirkungen technologischer und organisatorischer Veränderungen auf institutionelle Gegebenheiten (wie z. B. Veränderungen der Tarifbindung) und auf Sonderfaktoren (wie z. B. Arbeitskräftemobilität aufgrund von Grenzöffnungen) zurückzuführen sein.

Die vorliegenden empirischen Ergebnisse zur Entwicklung der Entlohnung und Beschäftigung bestätigen die Hypothese des nicht qualifikationsneutralen organisatorischen Wandels im wesentlichen für die Gruppe der besser qualifizierten Erwerbspersonen - vor allem für die Akademiker. Für die Un- und Angelernten lassen sich adverse Beschäftigungs- und Lohneffekte nicht mit betrieblichen Reorganisationsmaßnahmen in Verbindung bringen. Für eine Diagnose der Beschäftigungs- und Entlohnungssituation der Un- und Angelernten müssen deshalb weitere Erklärungsfaktoren herangezogen werden:

Erstens ist die Zuwanderung von Personen ohne oder ohne anerkannten Berufsabschluss aus Ost- und Mitteleuropa und Ostdeutschland nach Westdeutschland mit einer starken Erhöhung des Arbeitsangebots in den 90er Jahren in Verbindung zu bringen (Dustmann et al. 2009).

Hinzu kommt zweitens die abnehmende betriebliche Bindung an Flächentarifverträge, die auch zu einer Kompression der Verteilung der Arbeitseinkommen vor allem im unteren Teil der Verteilung geführt hat (Fitzenberger und Kohn 2005; Gerlach und Stephan 2005, 2006; Ellguth und Kohaut 2010a, 2010b). Dustmann et al. (2009) nutzen die LinkedEmployer-Employee-Daten des IAB, um die Lohnänderungen zwischen 1995 und 2004 für eine Dekompositionsanalyse durchzuführen. Sie finden heraus, dass bei einer Beibehaltung der betrieblichen Bindung an Flächentarifverträge, wie sie im Jahre 1995 bestanden hat, im Jahre 2004 die Löhne beim untersten 5-Prozent-Perzentil um 5,5 \% höher wären, aber beim 85 -Prozent-Perzentil nur um 0,2 \%.

Drittens ist die zunehmende Verbreitung neuerer Produktionskonzepte anzuführen (Abel et al. 2009; Zeller 2003, 2007; Bellmann und Stegmaier 2007, 2010). Damit einher gehen zunehmende Qualifikationsanforderungen bei Unund Angelernten, die Wartungs- und Qualitätssicherungsaufgaben übernehmen. Dies gilt auch für Personen mit Berufsabschluss, die aber nicht als Facharbeiter beschäftigt werden.
Viertens ist in den letzten Jahren die Idee des technologischen Wandels erneut aufgegriffen worden, wobei ein differenziertes Bild entworfen wurde: Während die Hypothese des „,skill-biased technological change“ von einem Beschäftigungszuwachs für die qualifizierten Beschäftigten und einem Beschäftigungsrückgang für die un- und angelernten Beschäftigten ausgeht, haben Autor et al. (2006), Goos und Manning (2007), und Goos et al. (2009) einen Beschäftigungsanstieg nicht nur bei Hochlohnarbeitsplätzen ermittelt, sondern auch bei schlechter bezahlten Arbeitsplätzen im Verarbeitenden Gewerbe und im Dienstleistungsbereich. Entscheidend ist aus ihrer Sicht die Möglichkeit der Gestaltung und Durchführung von Tätigkeiten als Routineaufgaben. Europaweit hat sich der Beschäftigtenanteil von Berufen wie z. B. Sicherheitspersonal, Verkäufer und Büroangestellte im Zeitraum 1993 bis 2006 deutlich erhöht (Goos et al. 2009). Empirische Evidenz für Deutschland präsentieren Dustmann et al. (2009).

\section{Wirtschaftspolitische Folgerungen}

Für die letzten drei Jahrzehnte lassen sich deutliche Veränderungen der qualifikatorischen Einkommensverteilung sowie der Beschäftigung, der Arbeitslosigkeit und der Erwerbstätigkeit Un- und Angelernter feststellen: Dabei hat sich die relative Position der Un- und Angelernten insgesamt im Vergleich zu den Qualifizierten verschlechtert. In unserm Projekt konnten zwar mit den Daten des IABBetriebspanels und den Sozialversicherungsdaten erstellten Linked-Employer-Employee-Daten des IAB die positiven Auswirkungen des organisatorischen Wandels für die qualifizierten Beschäftigten belegt werden. Allerdings konnten die negativen Beschäftigungs- und Lohnentwicklungen für die Un- und Angelernten nicht auf betriebliche Reorganisationsmaßnahmen zurückgeführt werden, obwohl alternative methodische Ansätze verwendet werden. Dieses Ergebnis ist kompatibel mit anderen empirischen Studien z. B. von Dustmann et al. (2009), die z. B. den Einfluss der Zuwanderung von Personen ohne oder ohne anerkannten Berufsabschluss aus Ost- und Mitteleuropa, die abnehmende Bindung an Flächentarifverträge, die Verbreitung neuerer Produktionskonzepte und die Gestaltung und Durchführung von Tätigkeiten als Routineaufgaben betonen.

Welche wirtschaftspolitischen Schlussfolgerungen lassen sich aus diesen Ergebnissen ziehen? Zunächst sind betriebliche Reorganisationsprozesse durch verschiedene Bündel von Maßnahmen, wie z. B. den Abbau von Hierarchieebenen, Gruppenarbeit, job rotation, Weiterbildung, Anreizentlohnung und flexiblen Arbeitszeiten gekennzeichnet. Die Verlagerung von Kompetenzen, Gruppenarbeit oder ein engerer Kundenkontakt erfordern Kommunikationsfähigkeit, Sozialkompetenz, Urteilsvermögen und neben Kreativität 
auch die Eigenschaft, mit anderen zusammenzuarbeiten. Diese Kompetenzen sollten insbesondere bei den Un- und Angelernten durch geeignete Weiterbildungsmaßnahmen, die vorzugsweise on-the-job stattfinden, gefördert werden. Dabei sind für Angehörige von Problemgruppen wie Ältere und Geringqualifizierte sowie für KMU öffentliche Förderprogramme wie das Sonderprogramm Weiterbildung gering Qualifizierter und beschäftigter „Älterer im Unternehmen“ (WeGebAU) der Bundesagentur für Arbeit sinnvoll (Bernhard et al. 2009). Darüber hinaus könnten die positiven Auswirkungen der Einführung der Gruppenarbeit auch Anlass zur Überlegung sein, Fördermittel z. B. für KMU zur Verfügung zu stellen, um diesen Unternehmen Reorganisationsmaßnahmen durch die Begleitung von Personalberatern zu erleichtern.

Im Bereich der lohnpolitischen Schlussfolgerungen ist die empirische Evidenz weniger eindeutig. Zunächst zeigen die Ergebnisse, dass indirekte Effekte von Reorganisationen vermittelt über die Entlohnung nur eine geringe Bedeutung für die Stabilisierung von Beschäftigungsverhältnissen haben. Dies bedeutet, dass von staatlicher Seite in diesem $\mathrm{Zu}$ sammenhang weniger an Lohnsubventionen zu denken ist, sondern mehr an die Förderung solcher Reorganisationsmaßnahmen, die beschäftigungsstabilisierend wirken. Dabei ist allerdings zu beachten, dass es kaum Reorganisationsmaßnahmen gibt, die gleichermaßen Löhne und Beschäftigung positiv beeinflussen, wie die empirischen Untersuchungen gezeigt haben.

Die Einführung von Gruppenarbeit kann von den Unternehmen als Instrument genutzt werden, um Beschäftigte im Betrieb zu halten, weil diese Reorganisationsmaßnahme sich v.a. bei besserqualifizierten Arbeitskräften nach unseren Untersuchungen als beschäftigungsstabilisierend erwiesen hat.

Allerdings sollten Unternehmen generell behutsam die Einführung von Reorganisationsmaßnahmen planen. Beispielsweise sollten sie sich nicht nur an den direkten positiven Produktivitätswirkungen orientieren, sondern auch die Bedürfnisse und mögliche Reaktionen ihrer Beschäftigten berücksichtigen. Dabei ist sicherlich die innerbetriebliche Diskussion und Kommunikation wichtig. In diesem Zusammenhang spielt die betriebliche Interessensvertretung und dabei insbesondere die Qualität der Zusammenarbeit mit der Unternehmensleitung eine wichtige Rolle.

Der Argumentation von Krugman (1994) folgend sind die Lohn- und Beschäftigungseffekte des technologischen und des organisatorischen Wandels als zwei Seiten derselben Medaille zu betrachten. Insofern könnte prinzipiell die Qualifikationsverzerrung des technisch-organisatorischen Wandels zu negativen (positiven) Lohn- und/oder Beschäftigungseffekten für un- und angelernte (qualifizierte) Beschäftigte führen. Unsere empirischen Ergebnisse belegen diese These allerdings nicht. Hingegen finden sich in der in diesem Beitrag erwähnten Studie von Addison et al. (2008) Hinweise darauf, dass Lohnrigiditäten in Deutschland im Zusammenhang mit einem „skill bias“ des technologischorganisatorischen Wandels zu Beschäftigungsproblemen für Un- und Angelernte geführt haben. Dagegen führen Dustmann et al. (2009) das Beschäftigungswachstum im Niedriglohnbereich auf die in den letzten Jahrzehnten gewachsene Ungleichheit der Arbeitseinkommen in Deutschland zurück.

\section{Executive summary}

Organizational change is characterized by different measures of business policy, work organization and personnel policy, e.g. delayering, teamwork, job rotation, further training and education, incentive payment and flexible working time. These measures work both, alone and in combination, on employment and remuneration of employees of different skill levels. Relocation of responsibilities, team work or close customer contact call for communication skills, social competence, judgment, initiative, creativity and even the individual capacity to work in teams. If these skills are rather attributed to highly qualified workers, an enterprise may be strived to establish a higher qualification level after an organizational change. Against this background the hypothesis seems to be justified to assume, that organizational change causes adverse employment effects for low skilled workers.

Based on linked employer-employee data of the Institute of Employment Research (IAB) Nuremberg, built with the IAB Establishment Panel and the employment statistics register, positive effects of organizational change on labour demand can be shown. No empirical evidence could be found for a skill bias caused by organizational change in this respect. The demand functions, deducted from the generalized "Leontief"-cost function, have been estimated for four skill levels as seemingly unrelated regressions (SUR). Our results indicate a significant influence of these variables, which measure reorganizational change. Evidence for a skill bias of reorganizational measures on the other hand could not be found.

Moreover positive effects on wages and stabilizing effects on employment appeared more often among middle or highly educated employees. This result corroborates the hypothesis of a skill biased organizational change. Clearly destabilizing effects on employment for low skilled workers could not be found in this study, despite using alternative methodological approaches.

This result is compatible to more recent studies concerning job polarization and those, which show an increasing diffusion of new production concepts coming along with tasks in maintenance service and quality assurance for low skilled workers. Furthermore, the large inflow of relatively low-skilled East Germans, Eastern Europeans, and ethnic 
Germans from Eastern Europe into the West German labor market and the remarkable decline in the share of workers covered by branch-level agreements should be taken into consideration.

Conclusions for labour market policy include the payment for subsidies for firms which offer further training and/or adopting teamwork, because these organizational measures tend to improve firm's competitiveness and employment level. Since we only find weak empirical evidence for direct and indirect effects of organizational measures on wages, the payment of wage subsidies is not the recommended strategy. Furthermore, establishments should take care of the employees' interests during the process of reorganization.

Danksagung Der Autor dankt der DFG für die Förderung des Projekts „Beschäftigungs- und Lohnwirkungen von betrieblichen Reorganisationsprozessen“ (im Rahmen des Schwerpunktprogramms „Flexibilisierungspotentiale bei heterogenen Arbeitsmärkten“).

\section{Literatur}

Abel, J., Hirsch-Kreinsen, H., Ittermann, P.: Einfacharbeit in der Industrie: Status Quo und Entwicklungsperspektiven. Soziologisches Arbeitspapier Nr. 24 der Technischen Universität Dortmund (2009)

Addison, J.T., Bellmann, L., Schank, Th., Teixeira, P.: The demand for labor. An analysis using matched employer-employee data from the German LIAB. Will the high unskilled worker own-wage elasticity please stand up? J. Labor Res. 29(2), 114-137 (2008)

Alda, H., Bellmann, L.: Organisatorische Änderungen und betriebliche Beschäftigungs- und Qualifikationseffekte 1999-2001. Mitt. Arb.markt- Berufsforsch. 35, 523-545 (2002)

Aoki, M.: A new paradigm of work organization and coordination? Lessons from the Japanese experience. In: Marglin, S. (Hrsg.) The Golden Age of Capitalism, S. 267-315. Wider Institute, Helsinki (1990)

Appelbaum, E., Bott, R.: The American Workplace. Cornell University Press, New York (1994)

Arellano, M., Bond, S.: Some test of specification for panel dam: Monte Carlo evidence and an application to employment equations. Rev. Econ. Stud. 58, 277-297 (1991)

Autor, D.H., Katz, L.F., Kearney, M.S.: The polarization of the U.S. labor market. Am. Econ. Rev. Pap. Proc. 96, 189-194 (2006)

Autorengruppe Bildungsberichterstattung: Bildung in Deutschland. Bielefeld (2010)

Bauer, T.K., Bender, St.: Technological change, organizational change, and job turnover. Labour Econ. 11, 295-291 (2004)

Beckmann, M.: Unternehmenspolitik, Managerkontrolle und Personalabbau in Deutschland. Mitt. Arb.markt- Berufsforsch. 33(4), 594 608 (2000)

Bellmann, L., Kohaut, S.: Betriebliche Beschäftigungsentwicklung und Innovationsaktivitäten. Mitt. Arb.markt- Berufsforsch. 32(4), 416-422 (1999)

Bellmann, L., Kölling, A.: Technology, wages and churning in Western Germany. In: Laaksonen, S. (Hrsg.) The Evolution of Firms and Industries, Proceedings of the International Conference on Comparative Analysis of Enterprise Data, S. 417-429. Statistics Finland, Helsinki (1997)

Bellmann, L., Pahnke, A.: Auswirkungen organisatorischen Wandels auf die betriebliche Arbeitsnachfrage. Zeitschrift für Arbeitsmarktforschung 39(2), 201-233 (2006)
Bellmann, L., Schank, Th.: Innovations, wages and demand for heterogeneous labor. New evidence from a matched employeremployee-dataset. IAB discussion paper No. 6 (2000)

Bellmann, L., Stegmaier, J.: Einfache Arbeit in Deutschland: RestgröBe oder relevanter Beschäftigungsbereich? Perspektiven der Erwerbsarbeit: Einfache Arbeit in Deutschland, S. 10-24. FriedrichEbert-Stiftung, Bonn (2007)

Bellmann, L., Stegmaier, J.: Perspektiven der Erwerbsarbeit: Facharbeit in Deutschland. Dokumentation der Expertengespräche. Gesprächskreis Arbeit und Qualifizierung. Friedrich-Ebert-Stiftung, Bonn (2010)

Bellmann, L., Caliendo, M., Hujer, R., Radić, D.: Beschäftigungswirkungen des technisch-organisatorischen Wandels. Eine mikroökonometrische Analyse mit dem Linked IAB-Panel. Mitt. Arb.markt- Berufsforsch. 35, 297-314 (2002)

Bellmann, L., Cornelißen, Th., Hübler, O., Pahnke, A.: Betriebliche Reorganisation, Entlohnung und Beschäftigungsstabilität. Zeitschrift für Arbeitsmarktforschung 41(2-3), 259-285 (2008)

Bernhard, S., Hohmeyer, K., Jozwiak, E., Koch, S., Kruppe, Th., Stephan, G., Wolff, J.: Aktive Arbeitsmarktpolitik in Deutschland und ihre Wirkungen. In: Möller, J., Walwei, U. (Hrsg.) Handbuch Arbeitsmarkt 2009. IAB-Bibliothek, Bd. 314, S. 149-201 (2009)

Biersack, W., Kettner, A., Reinberg, A., Schreyer, F.: Akademiker/innen auf dem Arbeitsmarkt: Gut positioniert, gefragt und bald sehr knapp. IAB-Kurzbericht 18/2008. Nürnberg (2008)

Breshnahan, T.F., Brynjolfsson, E., Hitt, L.M.: Information technology, workplace organization, and the demand for skilled labor: firmlevel evidence. Q. J. Econ. 117(1), 339-376 (2002)

Caroli, E., van Reenen, J.: Skill-biased organizational change? Evidence from a panel of british and french establishments. Q. J. Econ. 116, 1449-1492 (2001)

Dustmann, C., Ludsteck, J., Schönberg, U.: Revisiting the German wage structure. Q. J. Econ. 124(2), 843-881 (2009)

Ellguth, P., Kohaut, S.: Tarifbindung und betriebliche Interessensvertretung. Aktuelle Ergebnisse aus dem IAB-Betriebspanel 2009. WSI-Mitt. 63(4), 204-209 (2010a)

Ellguth, P., Kohaut, S.: Auf der Flucht? Wann Betriebe den Branchentarif verlassen. Ind. Bezieh. 17(4), 345-371 (2010b)

Fitzenberger, B., Kohn, K.: Gleicher Lohn für gleiche Arbeit? Zum Zusammenhang zwischen Gewerkschaftsmitgliedschaft und Lohnstruktur in Westdeutschland 1985-1997. J. Labour Mark. Res. 38 125-146 (2005)

Gallie, D., White, M., Chen, Y., Tomlinson, M.: Restructuring the Employment Relationship. Clarendon Press, Oxford (1998)

Gerlach, K., Stephan, G.: Wage settlements and wage setting-results from a multi-level model. Appl. Econ. 37, 2297-2306 (2005)

Gerlach, K., Stephan, G.: Bargaining regimes and wage dispersion. Jahrb. Natl.ökon. Stat. 226, 629-645 (2006)

Goos, M., Manning, A.: Lousy and lovely jobs: the rising polarization of work in Britain. Rev. Econ. Stat. 89, 118-133 (2007)

Goos, M., Manning, A., Salomons, A.: Job polarization in Europe. Am. Econ. Rev. Pap. Proc. 99, 58-63 (2009)

Greenan, N., Guellec, D.: Firm organization, technology and performance: an empirical study. Econ. Innov. New Technol. 6, 313-347 (1998)

Hammer, M., Champy, J.: Reengineering the Corporations. Harper Business, New York (1993)

Hujer, R., Caliendo, M., Radić, D.: Skill biased technological and organizational change: estimating a mixed simultaneous equation model using the IAB establishment panel. IZA discussion paper No. 566, Bonn (2002)

Kirchner, St., Oppen, M., Bellmann, L.: Zur gesellschaftlichen Einbettung von Organisationswandel. IAB-Diskussionspapier 37, Nürnberg (2008)

Kölling, A., Schank, Th.: Skill-biased technological change, international trade and the wage structure. Friedrich-AlexanderUniversität Erlangen-Nürnberg, Lehrstuhl Volkswirtschaftslehre/ 
Arbeitsmarkt- und Regionalpolitik, Discussion paper No. 14 (2002)

Krugman, P.: Past and prospective causes of unemployment: economic review. Federal Reserve Bank of Kansas City, QIV, S. 23-43 (1994)

Lindbeck, A., Snower, D.J.: Reorganization of firms and labor-market inequality. Am. Econ. Rev. Pap. Proc. 86, 315-321 (1996)

Lindbeck, A., Snower, D.J.: Multitask learning and the reorganization of work: from tayloristic to holistic organization. J. Labor Econ. 18(3), 353-376 (2000)

Osterman, P.: How common is workplace transformation and who adopts it? Ind. Labor Relat. Rev. 47(2), 173-189 (1994)

Pfeiffer, J.: Competitive Advantage Through People. Harvard Business School Press, Boston (1994)

Piva, M., Santarelli, E., Vivarelli, M.: The skill bias effect of technological and organisational change: evidence and policy implications. IZA discussion paper No. 934, Bonn (2003)

Zeller, B.: Trends der Qualifikationsentwicklung für einfache Tätigkeiten: Dienstleistung in komplexen Strukturen. Beispiel: Logistik. In: Bullinger, H.-J., Lidion, G., Schnalzer, K. (Hrsg.) Transport in die Zukunft - Berufliche Entwicklungen in Logistik und E-Commerce, S. 115-126. Bielefeld, Bertelsmann (2003)

Zeller, B.: Neue Qualifikationsanforderungen an der Schnittstelle von einfacher Arbeit und Facharbeit am Beispiel der Elektroindustrie.
In: Dietrich, H., Severing, E. (Hrsg.) Zukunft der dualen Berufsausbildung - Wettbewerb der Bildungsgänge. Schriften zur Berufsbildungsforschung der Arbeitsgemeinschaft Berufsbildungsforschungsnetz, S. 61-82. Bielefeld, Bertelsmann (2007)

Prof. Dr. Lutz Bellmann Studium der Wirtschaftswissenschaften, 1980 Abschluss als Diplom-Ökonom, danach wissenschaftlicher Mitarbeiter am Institut für Quantitative Wirtschaftsforschung der Universität Hannover und 1985 Promotion zum Dr. rer. pol., 2003 Habilitation an der Universität Hannover. Seit 1988 im Institut für Arbeitsmarktund Berufsforschung (IAB). Leitung des IAB-Betriebspanels seit 1997 und seit 2000 des Forschungsbereichs „Betriebe und Beschäftigung“ des IAB. Seit 2009 Inhaber des Lehrstuhls für Volkswirtschaftslehre, insbesondere Arbeitsökonomie an der Universität ErlangenNürnberg. Lehrtätigkeiten an den Universitäten Bamberg, Basel, Hannover, Passau, Speyer, Trier und Würzburg. Research Fellow des Instituts Zukunft der Arbeit.

Forschungsfelder: Arbeitsmarktökonomik, insbesondere Lohnstruktur, Beschäftigungsdynamik und der betrieblichen Aus- und Weiterbildung sowie Mikroökonometrie. 